ABNORMAL BREATHING PATTERNS AND HYPOXEMIA ASSOCIATED WITII FEEOING BEYOND THE NEONATAL PERIOD Karl H. P. Bentele*, Michael Albani and F. J. Schult Department of Pediatrics, University of Hamburg, FRG In order to assess the occurrance of feeding related abnormal breathing patterns and hypoxemia beyond the neonatal period polygraphic recordings incl.tcp0 were obtained in 23 infants during feeding and sleep also: 18 preterms, gest.age $30(27-36)$ wks., 14 after resp.distress syndrom(ROS) and 4 af ter apnoea of prematurity $(A O P)$ as well as 5 fullterms after a life threatening event related to feeding in 4 infants, postnatal age 1-13 wks.

Results: In preterms, feeding related decreases of tcpo (up to $80 \%$ ) were found in $12(66 \%)$, bradycardia and abnormal breathing patterns (period.apnoea(4), mixed apnoes (2), cyanosis(2)) as well as abnormal sleep apnoea (100\% period.breathing(2), obstructive \& mixed apnoea(4)) in 6 of them(50\%). Postnatal age in infants with and without feeding hypoxemia was 10 and 12.5 wks. respectively. In fullterms, decrease of tcpo under feeding (up to 22\%) occurred in 4, resp. dysrhythmia in 1 and abnormal sleep apnoea in 3: excessive period.breathing(2), obstr.\& mixed apnoea(1). Consequences were: tube feeding(5), more frequent feedings(7) with monit.tcpo, suppl. oxygen during feedings( 1 ). In infants with tcp $0<40 \mathrm{~mm} \mathrm{Hg}$ abnormal findings had disappeared within 2-5 wks. Conclusions: preterms ${ }^{2}$ recovering from ROS or AOI may have feeding related hypoxemia and resp.pattern abnormalities even beyond 38 wks.conc.age, which will not be detected withoutmonitoring $\mathrm{p}_{2}$ (or $0_{2}$ sat.). These findings seem to reflect a developmental deficit in neuronal coordination of breathing and feeding, which under profound hypoxemia may progress to asphyxia. and cause residual morbidity. (*) supported by ofG grant Be $921 / 1-3$
40 NEONATAL AUDITORY BRAYNSTEM RESPONSES (ABR) PREDICT SENSORY NEURAL HEARING LOSS (SNHL) AT 4 YEARS.

BC.Bradford, JWP.Hazell, A.Stewart, EOR.Reynolds.

Departments of Paediatrics and Audiology, University College and Middlesex School of Medicine, London, England.

To find out if $A B R$ elicited in the neonatal period predicted later hearing status, 117 very preterm ( $<33$ weeks) infants who were tested shortly after birth had hearing assessments at age 4 years. The ABR tests were made with an Amplaid Mk IV in response to wideband high frequency clicks at $100 \mathrm{~dB}$ peak equivalent sound pressure level at gestational equivalent ages of 30-42 weeks. Infants with absent responses were re-tested at 3 months. All children had a puretone audiogram at 4 years of age; those with abnormal audiograms had a full oto-audiological investigation. 103 children had $A B R$ in the neonatal period; 101 had normal hearing at 4 years, 1 had a moderate high tone loss and 1 a unilateral loss at 2 $\mathrm{kHz}$, only. ABR were absent in the neonatal period in 14 children. Responses were present at 3 months in 4 children; 3 of the 4 had normal hearing and 1 a loss at $4 \mathrm{kHz}$ only. All 10 children who still had absent ABR at 3 months had SNHL; 9 of the 10 required active treatment. We conclude that neonatal ABR accurately identify very preterm infants who have SNHL which requires active treatment. \section{HAND EYE COORDINATION IN 7-YEAR OLD LOW BIRTHWEIGHT}

Alison, A Ellinan, Elizabeth M. Bryan, Anthony D.

Hammersmith and Queen Charlotte's Hospitals, London Brunel University, Uxbridge, UK.

As part of a larger study, hand-eye coordination was measured in a group of low birthweight (LBW) (Bir<2000g) 7 year olds attending normal school, and a control group of normal birthweight children selected from local schools. Children with cerebral palsy were excluded. The distribution of sex, social class and race was similar in the two groups but there were significantly more first surviving and only children in the IBBW grous $(p<.005)$. LBW children were less good at finger-nose pointing $(p<.01)$, diadochokinesis $(p<<.001)$ and finger opposition using both hands simultaneously $(p<.05)$. There was no significant difference in the ability to throw a bail up and catch it, but LBW children were less able to clap while the ball was in the air (p<.001). Within the LBW group there was no significant difference between children of different birth weight, sex or gestation, but children who were appropriate for gestational age (AGA) were better at diadochokinesis than those who were small for gestational age $(p<.05)$. These findings show that LBW children are less good at hand-eye coordination than children of normal birthweight.
39 ABNORMAL COGNITIVE AND PROCESSING SKILLS IN VERY PRETERM INFANTS AT 8 YEARS OF AGE. J Baudin, B Lloyd, D Edwards, J Townsend, A Stewart, EOR Reynolds. Dept of Paediatrics, University college and Middlesex School of Medicine, London WCIE 6JJ. and Middlesex School of Medicine, London WC1E $6 \mathrm{JJ}$. ultrasound scan findings and later outcome in very preterm $\quad(<33$ weeks) infants, cognitive and processing skills were tested at 8 years. 54 children were tested with the WISC-R and the Kaufman Assessment Battery for Children ( $K \rightarrow A B C$ ) in the course of a detailed assessment. The mean WISC IQ was $103 \pm$ sd 15. 24 $(44 \%)$ of the children had a significant ( $<<0.05)$ subscale difference, including 16 where the verbal score was greater difference, including 16 where the verbal score was greater
than the performance score. The mean Kaufman Mental Processing than the performance score. The mean Kaufman Mental Processing
composite was $110 \pm$ sd 13 which was significantly greater $(p<0.001)$ than the mean WISC IQ. $30(56 \%)$ of the children had $\mathrm{K}-\mathrm{ABC}$ processing scores which differed significantly, including 25 at the $1 \%$ level. In 26 of the 30 children the difference was in favour of sequential processing. These results suggest interference with right hemisphere function. There was no relation between simultaneous processing deficit and neonatal ultrasound brain scan findings. We speculate that and neonatal ultrasound brain scan findings. We speculate that for the learning failure often reported in very preterm infants.
41 PREDICTION BY NUCLEAR MAGNETIC RESONANCE SPECTROSCOPY (MRS) OF NEURODEVELOPMENTAL OUTCOME IN INFANTS WITH INCREASED CEREBRAL ECHODENSITIES. D. Azzopardi, JS.Wyatt, J.Baudin, EB.Cady, DT.Delpy, Paediatrics, University College and Middlesex School of Medicine, London, WC1E 6JJ. England.

The aim of this study was to investigate the prognostic significance of abnormalities of cerebral oxidative phosphorylation in infants with increased echodensities. 46 newborn infants who developed increased echodensities were studied by MRS. Neurodevelopmental outcome was assessed in the survivors at 12 months of corrected age. 19 of the 27 infants with values for phosphocreatine $(\mathrm{PCr}) /$ inorganic orthophosphate (Pi) below $95 \%$ confidence limits for normal in survivors developed disabling neurodevelopmental impairments and 7 had developmental quotients (DQ) below 80 . By contrast only 2 of 19 infants with normal values for $\mathrm{PCr} / \mathrm{Pi}$ died and although 10 had impairments at 12 months their disabilities were less severe and the DO was 80 or above in 15 infants.

We conclude: 1. Definite abnormalities of oxidative phosphorylation indicated severe brain damage and a very poor prognosis. 2. Some infants with apparently normal oxidative phosphorylation developed less severe brain damage which had phosphorylation developed less severe
not been detected by surface-coil MRS.

42 IMPACT OF A DECLINING LOW BIRTHWEIGHT RATE ON PERINATAL MORTALITY AND INCIDENCE OF CEREBRAI PALSY

Alf Meberg, spon. by Asbjørn Langslet Department of Paediatrics, Vestfold Central Hospital, Tønsberg, and the Medical Birth

Registry, University of Bergen, Norway

In infants $500 \mathrm{~g}$ or more born in a Norwegian County (Vestfold) 1970-84 ( $\mathrm{n}=34756)$, a significantly declining incidence of low birthweight (500-2499 g) occurred, from $5 \% 1970-79$ to 4.18 1980-84 (p<0.01). The decline was stronger for infants 500-1499 g than for infants 1500$2499 \mathrm{~g}(31 \%$ vs. $138 ; \mathrm{p}<0.01)$. Perinatal mortality decreased from 17 to $10.30 / 00(\mathrm{p}<0.01)$, and the incidecreased from 17 to $10.30 / 00(\mathrm{p}<0.01)$, and the
dence of cerebral palsy (CP) from 2.8 to $1.70 / 00$ $(p=0.07)$. Low birthweight infants were at increased risk for perinatal death and CP. Thus, $38 \%$ of the'decline in perinatal mortality and 18.28 of the reduction of $\mathrm{CP}$ were attributable to the decline in low birthweight incidence. Changes in distribution of birthweights may be corrected for when quality of perinatal care is to be evaluated. Strategies for prevention of low birthweight may be important for reduction of perinatal mortality and handicaps related to low birthweight. 